\title{
Predicting insulin resistance using the triglyceride-to-high-density lipoprotein cholesterol ratio in Taiwanese adults
}

\author{
Jui-Kun Chiang ${ }^{1,2}$, Ning-Sheng Lai ${ }^{3,4}$, Jiunn-Kae Chang $^{2}$ and Malcolm Koo ${ }^{5^{*}}$
}

\begin{abstract}
Background: The triglyceride to high-density lipoprotein cholesterol ratio (TG/HDL-C) has been advocated as a simple clinical indicator of insulin resistance. Thresholds of TG/HDL-C appeared to depend on ethnicity. However, no studies have specifically compared the accuracy of TG/HDL-C with and without other clinical and demographic factors in predicting insulin resistance in Taiwanese adults. The aim of the present investigation was to use TG/ HDL-C and other clinical available factors to predict insulin resistance in Taiwanese adults.

Methods: A total of 812 subjects were recruited from at the time of their general health examination at the Buddhist Dalin Tzu Chi General Hospital, Taiwan. Demographic information and clinical characteristics were obtained. Insulin resistance was defined by the homeostasis model assessment for insulin resistance (HOMA-IR). Simple and multiple logistic regression analyses were used to obtain probabilities of insulin resistance (HOMA-IR > 2) using TG/HDL-C with (Model 2) and without (Model 1) other clinical variables. A receiver operating characteristic (ROC) analysis was conducted to evaluate the ability of the two models to correctly discriminate between subjects of low and elevated HOMA-IR.
\end{abstract}

Results: Female sex, greater waist circumferences, and higher ALT levels were significantly associated with the risk of elevated HOMA-IR in addition to TG/HDL-C in the multiple logistic regression (Model 2). The area under the ROC curve (AUC) of Model 2 was $0.71[95 \% \mathrm{Cl}=0.67-0.75]$ and was significantly higher $(P=0.007)$ than the AUC 0.66 [95\% $\mathrm{Cl}=0.62-0.71]$ of Model 1 .

Conclusions: The diagnostic accuracy of insulin resistance, defined by HOMA-IR, using TG/HDL-C can be significantly enhanced by including three additional clinically available factors - sex, waist circumferences, and ALT levels.

Keywords: prediction, insulin resistance, Chinese, ethnic groups

\section{Background}

Metabolic syndrome is characteristically defined as a clustering condition of cardiovascular risk factors including hyperglycemia, dyslipidemia, hypertension, and central obesity [1]. Its occurrence is strongly associated with increased risk in the development of diabetes and cardiovascular disease. A recent meta-analysis of 87 prospective observational studies reported that metabolic syndrome was associated with a two-fold increase

\footnotetext{
* Correspondence: m.koo@utoronto.ca

${ }^{5}$ Dalla Lana School of Public Health, University of Toronto, Ontario, Canada Full list of author information is available at the end of the article
}

in cardiovascular disease outcomes and a 50\% increase in risk of all-cause mortality [2].

The pathophysiology of the metabolic syndrome remains a subject of controversy but many of its features are associated with insulin resistance. It is typically defined as decreased sensitivity or responsiveness to metabolic actions of insulin. Currently, there are several directly and indirect methods to assess insulin resistance [3]. The gold standard method was the hyperinsulinemic-euglycemic clamp (glucose clamp) technique, originally developed by DeFronzo [4]. However, the method is complex and time consuming, making it not feasible for either epidemiological investigations or

\section{C) Biomed Central}


routine clinical applications. Therefore, a number of surrogate indices of insulin resistance have been developed. Previous studies have shown that insulin resistance scores based on the homeostasis model assessment (HOMA) method was strongly correlated with glucose clamp-assessed insulin resistance [5,6]. The model utilize a set of empirically derived nonlinear equations to predict the homeostatic concentrations of fasting insulin and glucose, which reflect the varying degrees of pancreatic $\beta$-cell function and insulin resistance [7].

Although HOMR-IR has been widely used in the study of metabolic syndrome, the lack of standardized insulin assay has limited its clinical utility [8]. It would be of considerable benefit for clinicians if other standardized measures are available for predicting insulin resistance without the need for direct measurements of fasting insulin. Since hypertriglyceridemia and low highdensity lipoprotein cholesterol are two key metabolic abnormalities associated with insulin resistance states $[9,10]$, the triglyceride to high-density lipoprotein cholesterol ratio (TG/HDL-C) has been advocated as a simple clinical indicator of insulin resistance [11,12]. It has been evaluated as predictor of diabetes [13] and coronary heart disease [14]. In a cohort study on 3,014 patients, the area under the receiver operating characteristic curves (AUC) for predicting insulin resistance with TG/HDL-C was 0.75 [14]. Nevertheless, recent studies have reported that racial differences existed in the predictability of the ratio [15]. In a cross-sectional study of 50 white and 99 African American adults, the AUCs of TG/HDL-C for predicting insulin resistance, as measured by HOMA-IR, was found to be 0.77 and 0.64 , respectively. It was concluded that the relationship between TG/HDL-C and insulin resistance varied in different ethnic groups and TG/HDL-C was not a reliable marker for predicting insulin resistance in African Americans [16]. On the other hand, a cross-sectional study of 6,546 Korean adults who underwent routine health examinations found that TG/HDL-C was a consistent indicator of insulin resistance in subjects without metabolic syndrome [17].

In addition, depending on ethnicity, different thresholds of TG/HDL-C have been proposed for predicting insulin resistance. A ratio of 3.0 was suggested for nonHispanic whites and Mexican Americans, 2.0 for nonHispanic blacks [18], and 2.5 for African-American men [19]. Despite of numerous studies have evaluated the optimal threshold for TG/HDL-C, to the best of our knowledge, no studies have specifically compared the accuracy of TG/HDL-C with and without other clinical and demographic factors in predicting insulin resistance. Moreover, no studies have reported that prediction of insulin resistance using TG/HDL-C in Taiwanese adults. Hence, the aim of this study was to evaluate the prediction of insulin resistance by TG/HDL-C with and without the inclusion of other clinical available factors for clinical applications.

\section{Methods}

\section{Study Subjects}

Participants were recruited at the time of their general health examination at the Buddhist Dalin Tzu Chi General Hospital located in Chiayi county, Taiwan between May 2007 and April 2008. The study hospital is located in south Taiwan and most of the participants came from Yulin, Chiayi, Tainan, and Kaoshiung counties in southern Taiwan.

This study was approved by the Institutional Review Board of the study hospital and written informed consent was given by all the participants before enrollment.

Information on demographic and clinical characteristics including age, sex, location of residence, weight, height, waist circumference, and sitting blood pressure were collected. Body mass index was calculated by dividing weight (in kilograms) by the square of the height (in meters). Daily energy expenditure was estimated using a seven-day physical activity recall questionnaire (Chinese version) [20].

\section{Sample processing and analyses}

Triglyceride, total cholesterol, HDL-C, LDL-C, glucose, insulin, and alaninne aminotrasferase (ALT) were measured using blood sample collected from each subject after a minimum eight hour fasting period. Plasma fasting insulin concentrations were measured using human insulin Enzyme Linked Immunosorbent Assay (ELISA) kit (BioSource Europe S.A., Nivelles, Belgium). Insulin resistance was defined by homeostasis model assessment for insulin resistance (HOMA-IR). HOMAIR was calculated by dividing the product of fasting plasma glucose $(\mathrm{mg} / \mathrm{dL})$ and fasting plasma insulin (mU/L) by 405 [5]. Other biochemical variables including blood lipids, and glucose were analyzed using an auto-analyzer (Sysmex XE-2100 Blood Cell Analyzer, Kobe, Japan).

\section{Statistical Analyses}

Statistical analysis was performed using the $R$, version 2.12.1, software (Free Software Foundation, Inc., Boston, MA, U.S.A.). Subjects were divided into elevated HOMA-IR and low HOMA-IR groups using a cutoff value of 2 . Two-sided $p$ value $\leq 0.05$ was considered statistically significant. Continuous data were expressed as mean \pm standard deviation (SD) and categorical variables were represented by frequency and percentage. Differences in demographic and clinical characteristics of subjects between the low and elevated HOMA-IR groups were examined using the two-sample $t$ test for 
continuous variables and Fisher's exact test for categorical variables.

Simple logistic regression was used to obtain probability of insulin resistance (HOMA-IR > 2) using only TG/ HDL-C (Model 1). Multiple logistic regression analysis with stepwise variable selection procedure using the Akaike's Information Criterion (AIC) was also conducted to evaluate other factors that were significantly associated with insulin resistance in addition to TG/ HDL-C (Model 2). A ROC analysis was conducted to evaluate the ability of the two models for correctly discriminating the subjects of low and elevated HOMA-IR. Plots of the sensitivity (true positive) versus 1 -specificity (false positive) for the two models were made and the overall diagnostic accuracy between the two models was quantified using AUC. These two correlated ROC curves were then compared by applying the ROC test of DeLong using the roc.test function of the $p R O C$ library in $R$.

\section{Results}

A total of 812 subjects were recruited into the study after excluding 8 subjects using lipid-lowering medications, 53 subjects using antidiabetic medications, and 4 using both types of medications. Table 1 shows the demographic and clinical characteristics of subjects categorized by HOMA-IR using a cutoff value of 2 . The subjects comprised of 403 men aged $52.0 \pm 11.0$ [range $22.0-80.5$ ] years and 409 women aged $52.1 \pm 9.8$ [range 19.3-81.5]. There were no significant differences in age, sex, location of residence, body height, total cholesterol levels, low-density lipoprotein-cholesterol (LDL-C) levels, and daily energy expenditure between the two HOMA-IR groups. The mean body weight, body mass index, waist circumference, systolic blood pressure, diastolic blood pressure, fasting glucose level, triglyceride level, alanine transaminase (ALT) level, and insulin level were significantly higher in the elevated HOMA-IR group. The mean high density lipoprotein-cholesterol (HDL-C) level was significantly lower in the elevated HOMA-IR group.

Table 2 shows the two models of the association between TG/HDL-C and HOMA-IR. Model 1 contained only TG/HDL-C as a predictor and Model 2 contained covariates in addition to TG/HDL-C that were obtained from stepwise multiple logistic regression. Female sex, greater waist circumferences, higher ALT levels, and higher TG/HDL-C were significantly associated with the prevalence odds of elevated HOMA-IR. In Model 1, an increase of 1 unit in TG/HDL-C ratio was associated with an elevation in the insulin resistance by $31 \%$ while in Model 2, the corresponding in the TG/HDL ratio was associated with an $19 \%$ elevation in the odds. Figure 1 shows the ROC curves between the two models. The
Table 1 Demographic and clinical characteristics of subjects categorized by HOMA-IR ( $\mathbf{N}=\mathbf{8 1 2}$ )

\begin{tabular}{|c|c|c|c|}
\hline Variable & $\begin{array}{c}\text { HOMA-IR } \\
\quad \leq 2 \\
n=593\end{array}$ & $\begin{array}{c}\text { HOMA-IR } \\
>2 \\
n=219\end{array}$ & $P$ \\
\hline Age (years) & $51.8 \pm 10.3$ & $52.9 \pm 10.6$ & 0.177 \\
\hline \multicolumn{4}{|l|}{ Sex } \\
\hline Male & $290(48.9)^{\dagger}$ & $113(51.6)$ & 0.496 \\
\hline Female & $303(51.1)$ & $106(48.4)$ & \\
\hline \multicolumn{4}{|l|}{ Location of residence } \\
\hline Metropolitan & $240(40.5)$ & $90(41.3)^{\ddagger}$ & 0.835 \\
\hline Other & $353(59.5)$ & $128(58.7)$ & \\
\hline Body weight (kg) & $61.6 \pm 9.99$ & $66.4 \pm 12.4$ & $<0.001$ \\
\hline Body height $(\mathrm{cm})$ & $161.9 \pm 8.1$ & $161.8 \pm 8.0$ & 0.799 \\
\hline Body mass index $\left(\mathrm{kg} / \mathrm{m}^{2}\right)$ & $23.4 \pm 2.9$ & $25.3 \pm 3.8$ & $<0.001$ \\
\hline Waist circumference $(\mathrm{cm})$ & $76.9 \pm 8.3$ & $82.5 \pm 10.0$ & $<0.001$ \\
\hline Systolic blood pressure $(\mathrm{mmHg})$ & $125.3 \pm 18.8$ & $129.0 \pm 19.8$ & 0.014 \\
\hline $\begin{array}{l}\text { Diastolic blood pressure } \\
(\mathrm{mmHg})\end{array}$ & $73.6 \pm 12.1$ & $76.6 \pm 12.0$ & 0.002 \\
\hline Fasting glucose (mg/dL) & $88.5 \pm 10.0$ & $93.6 \pm 11.7$ & $<0.001$ \\
\hline Triglyceride (mg/dL) & $102.9 \pm 59.5$ & $137.1 \pm 79.9$ & $<0.001$ \\
\hline Total cholesterol (mg/dL) & $192.7 \pm 34.4$ & $192.6 \pm 38.2$ & 0.968 \\
\hline $\mathrm{HDL}-\mathrm{C}(\mathrm{mg} / \mathrm{dL})$ & $56.3 \pm 14.8$ & $50.5 \pm 14.3$ & $<0.001$ \\
\hline $\mathrm{LDL}-\mathrm{C}(\mathrm{mg} / \mathrm{dL})$ & $127.5 \pm 31.6$ & $128.4 \pm 35.3$ & 0.738 \\
\hline Insulin (mU/L) & $4.84 \pm 2.06$ & $15.66 \pm 10.32$ & $<0.001$ \\
\hline $\mathrm{ALT}(\mathrm{mg} / \mathrm{dL})$ & $24.2 \pm 15.8$ & $38.1 \pm 50.2$ & $<0.001$ \\
\hline $\begin{array}{l}\text { Energy expenditure (Kcal×103/ } \\
\text { day) }\end{array}$ & $2.96 \pm 1.43$ & $3.03 \pm 1.12$ & 0.517 \\
\hline
\end{tabular}

* Continuous variables were represented by mean \pm standard deviation.

† Categorical variables were expressed as number (percentage).

* One missing data for HOMA-IR > 2 in location of residence.

HDL-C: high-density lipoprotein-cholesterol

LDL-C: low-density lipoprotein-cholesterol

ALT: alanine transaminase

AUC of Model 2 was 0.71 [95\% CI $=0.67-0.75]$, which was significantly higher $(P=0.007)$ than the AUC 0.66 $[95 \% \mathrm{CI}=0.62-0.71]$ of Model 1 . The codes for calculating the probability of elevated insulin resistance using Model 2 in the R environment, Microsoft Excel, and OpenOffice Calc are provided in Appendix 1. Note that the model should only be applied to ranges within the observed values, i.e., waist circumference between 57.1 $\mathrm{cm}$ and $111.8 \mathrm{~cm}$, ALT between $8 \mathrm{mg} / \mathrm{dL}$ and $526 \mathrm{mg} /$ $\mathrm{dL}$, and TG/HDL-C ratio between 0.26 and 22.26.

\section{Discussion}

This study aimed to develop a simple predictive model as a clinical tool for evaluation of insulin resistance. In clinical settings, it would be useful to identify individuals with insulin resistance using only routinely collected blood test results such as triglyceride and high-density lipoprotein cholesterol. Hence, we proposed the prediction of insulin resistance by the ratio of TG and HDL-C. 
Table 2 Two logistic regression models for predicting insulin resistance (HOMA-IR > 2)

\begin{tabular}{|c|c|c|c|c|c|}
\hline Variable & $\beta$ & $\begin{array}{l}\text { Standard } \\
\text { error }\end{array}$ & $P$ & Odds ratio & $95 \%$ confidence interval \\
\hline \multicolumn{6}{|l|}{ Model 1} \\
\hline Intercept & -1.642 & 0.135 & $<0.001$ & & \\
\hline $\mathrm{TG} / \mathrm{HDL}-\mathrm{C}$ & 0.257 & 0.042 & $<0.001$ & 1.29 & $1.19-1.41$ \\
\hline \multicolumn{6}{|l|}{ Model 2} \\
\hline Intercept & -7.941 & 0.986 & $<0.001$ & & \\
\hline \multicolumn{6}{|l|}{ Sex } \\
\hline Male & 1 & & & & \\
\hline Female & 0.933 & 0.207 & $<0.001$ & 2.54 & $1.70-3.84$ \\
\hline Waist circumference $(\mathrm{cm})$ & 0.071 & 0.012 & $<0.001$ & 1.07 & $1.05-1.10$ \\
\hline $\operatorname{ALT}(\mathrm{mg} / \mathrm{dL})$ & 0.015 & 0.004 & $<0.001$ & 1.01 & $1.01-1.02$ \\
\hline $\mathrm{TG} / \mathrm{HDL}-\mathrm{C}$ & 0.173 & 0.044 & $<0.001$ & 1.19 & $1.09-1.30$ \\
\hline
\end{tabular}

TG/HDL-C: triglyceride to high-density lipoprotein cholesterol ratio

ALT: alanine transaminase

One unique finding in this study was that we evaluated the accuracy of prediction of insulin resistance using TG/HDL-C with or without the presence of other readily available demographic and biochemical variables. The model that included three factors in addition to TG/HDL-C significantly increased the predicting probability of higher insulin resistance compared with the model containing only TG/HDL-C. The three additional factors were sex, waist circumferences, and ALT levels, which are readily available measurements in clinical settings.

Previous studies had used different cutoff values of TG/HDL-C to predict insulin resistance. However, the results were inconclusive. The cutoff point for TG/ HDL-C appeared to vary among different ethnic groups. It was reported to be 3.0 for non-Hispanic whites and

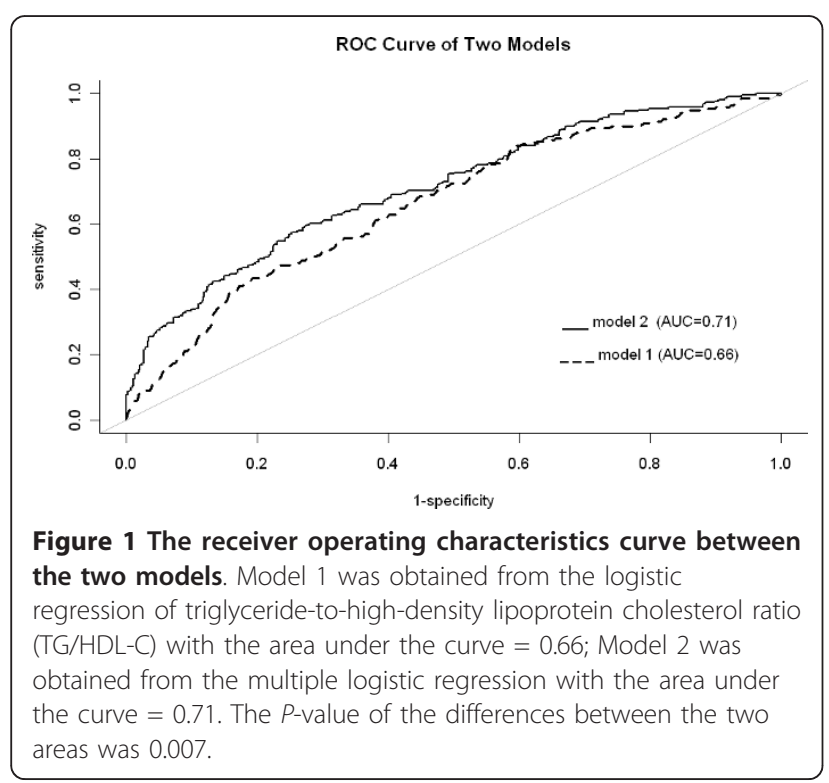

Mexican Americans and 2.0 for non-Hispanic blacks [18]. In contrast to these studies, the present study did not use a cutoff point for TG/HDL-C to predict insulin resistance. Instead, we treated TG/HDL-C as a continuous variable to predict the probability of insulin resistance as measured by HOMA-IR. In a study of 1,824 Iranian men of age 40 years and above, it was reported that the prevalence of metabolic syndrome in subjects with TG/HDL-C greater or equal to 6.9 was $63.6 \%$, compared with $3.0 \%$ in those with TG/HDL-C below 2.8 [21]. The risk of metabolic syndrome appeared to be incrementally associated with TG/HDL-C and there are no consensual cutoff values for it. Therefore, no cutoff point was set for TG/HDL-C in the present study.

In addition to the potential use as a predictor of insulin resistance in clinical settings, the ratio of TG and HDL-C has also been reported as a strong predictor of incident hypertension in a prospective study of 2831 non-hypertensive Middle Eastern women [22]. In addition, based on the findings from a study of 585 males with type 2 diabetes mellitus, $\log (\mathrm{TG}) / \mathrm{HDL}-\mathrm{C}$ was suggested to be used as a simple means of estimating atherogenic dyslipidemia, which is closely associated with the future risk of coronary artery disease [23]. Moreover, in a prospective study of a nationally representative sample of United States adults with diagnosed diabetes, higher serum non-HDL-c concentrations was found to be associated with increased risk of death from cardiovascular diseases [24]. In our study, higher serum non-HDL-c levels were directly and significantly correlated with levels of TG/HDL-C, with a Pearson correlation coefficient of 0.34 .

There are potential limitations regarding the interpretation of our results. First, previous research found that ethnicity affects the associations between TG/HDL-C and insulin resistance. The accuracy of the obtained 
model when apply to other ethnic groups will need additional evaluation. Second, the applicability of the model is limited to the observed data range of the present study.

\section{Conclusions}

Insulin resistance is the core of the metabolic syndrome and a pre-diabetes condition. The present study demonstrated that the diagnostic accuracy of insulin resistance, defined by HOMA-IR, using TG/HDL-C can be significantly enhanced in the prediction model by including sex, waist circumference, and ALT, which are readily available clinical measurements. The model described in this report could be served as a tool to assist health care professionals to easily and accurately predict insulin resistance and thereby providing timely lifestyle modifications advice to susceptible individuals.

\section{Appendix 1}

Programming code in R, Microsoft Excel and OpenOffice Calc for calculating the probability of elevated insulin resistance (HOMA-IR > 2) based on the multiple logistic regression model.

\section{In R environment}

To calculate the probability of insulin resistance (HOMA-IR > 2), substitute the values for the variables $\mathrm{X}_{1}$ to $\mathrm{X}_{4}$ in the following regression equation:

$$
\begin{aligned}
& \text { yhat }<-(-7.941 \\
& +0.933 * \mathrm{X}_{1} \\
& 1 \text {, male }=0 \text { ) } \\
& +0.071^{*} \mathrm{X}_{2} \\
& \text { ference }(\mathrm{cm}) \\
& +0.015^{*} \mathrm{X}_{3} \\
& +0.173^{*} \mathrm{X}_{4} \\
& \text { ) } \\
& \text { phat }<-1 /(\exp (-(\text { yhat }))+1) \\
& \text { phat } \\
& \text { \# constant } \\
& \# X_{1}=\operatorname{sex}(\text { female }= \\
& \# \mathrm{X}_{2}=\text { waist circum- } \\
& \# \mathrm{X}_{3}=\mathrm{ALT}(\mathrm{mg} / \mathrm{dL}) \\
& \# \mathrm{X}_{4}=\mathrm{TG} / \mathrm{HDL}-\mathrm{C} \\
& \text { \# copy these syntax }
\end{aligned}
$$

and paste on the $\mathrm{R}$ console

\section{In Microsoft Excel or OpenOffice Calc}

Key in the values for sex (female $=1$, male $=0$ ) in the A1 cell, waist circumference $(\mathrm{cm})$ in the A2 cell, ALT (mg/dL) in the A3 cell, and TG/HDL-C in the A4 cell.

Key in the following formula in any empty cell on the spreadsheet to obtain the probability of insulin resistance.

$$
=1 / \operatorname{EXP}(-(-7.941+0.933 * \mathrm{~A} 1+0.071 * \mathrm{~A} 2+0.015 * \mathrm{~A} 3+0.173 * \mathrm{~A} 4)+1)
$$

Note: The model should only be applied to individuals with waist circumference between $57.1 \mathrm{~cm}$ and 111.8 $\mathrm{cm}$, ALT between $8 \mathrm{mg} / \mathrm{dL}$ and $526 \mathrm{mg} / \mathrm{dL}$, and TG/ HDL-C between 0.26 and 22.26.

\section{Abbreviations}

HOMA-IR: homeostasis model assessment for insulin resistance; TG/HDL-C: triglyceride to high-density lipoprotein cholesterol ratio

\section{Acknowledgements}

This work was financially supported by the Buddhist Dalin Tzu Chi General Hospital (DTCRD 96-13, 97-18, 98 (2)-07, and 99(2)-E-05) to JK Chiang.

\section{Author details}

'Department of Family Medicine, Buddhist Dalin Tzu Chi General Hospital, Chiayi, Taiwan. ${ }^{2}$ Department of Biotechnology, Chia Nan University of Pharmacy \& Science, Tainan, Taiwan. ${ }^{3}$ Department of Allergy, Immunology and Rheumatology, Buddhist Dalin Tzu Chi General Hospital, Chiayi, Taiwan. ${ }^{4}$ School of Medicine, Tzu Chi University, Hualien, Taiwan. ${ }^{5}$ Dalla Lana School of Public Health, University of Toronto, Ontario, Canada.

\section{Authors' contributions}

JKC (CHIANG) analyzed the data and wrote the manuscript. NSL contributed to the discussion. JKC (CHANG) wrote the manuscript. MK edited the manuscript and contributed to the discussion. All authors read and approved the final manuscript

\section{Competing interests}

The authors declare that they have no competing interests.

Received: 6 October 2011 Accepted: 17 October 2011

Published: 17 October 2011

\section{References}

1. Alberti KG, Zimmet PZ: Definition, diagnosis and classification of diabetes mellitus and its complications. Part 1: diagnosis and classification of diabetes mellitus provisional report of a WHO consultation. Diabet Med 1998, 15:539-553.

2. Mottillo $S$, Filion KB, Genest J, Joseph L, Pilote L, Poirier P, Rinfret $S$, Schiffrin EL, Eisenberg MJ: The metabolic syndrome and cardiovascular risk a systematic review and meta-analysis. J Am Coll Cardiol 2010, 56:1113-1132

3. Muniyappa R, Lee S, Chen H, Quon MJ: Current approaches for assessing insulin sensitivity and resistance in vivo: advantages, limitations, and appropriate usage. Am J Physiol Endocrinol Metab 2008, 294:E15-26.

4. DeFronzo RA, Tobin JD, Andres R: Glucose clamp technique: a method for quantifying insulin secretion and resistance. Am J Physiol 1979, 237: E214-223.

5. Matthews DR, Hosker JP, Rudenski AS, Naylor BA, Treacher DF, Turner RC: Homeostasis model assessment: insulin resistance and beta-cell function from fasting plasma glucose and insulin concentrations in man. Diabetologia 1985, 28:412-419.

6. Bonora E, Targher G, Alberiche M, Bonadonna RC, Saggiani F, Zenere MB, Monauni T, Muggeo M: Homeostasis model assessment closely mirrors the glucose clamp technique in the assessment of insulin sensitivity: studies in subjects with various degrees of glucose tolerance and insulin sensitivity. Diabetes Care 2000, 23:57-63.

7. Wallace TM, Levy JC, Matthews DR: Use and abuse of HOMA modeling Diabetes Care 2004, 27:1487-1495

8. Miller WG, Thienpont LM, Van Uytfanghe K, Clark PM, Lindstedt P, Nilsson G, Steffes MW, Insulin Standardization Work Group: Toward standardization of insulin immunoassays. Clin Chem 2009, 55:1011-1018.

9. Lewis GF, Uffelman KD, Szeto LW, Steiner G: Effects of acute hyperinsulinemia on VLDL triglyceride and VLDL apoB production in normal weight and obese individuals. Diabetes 1993, 42:833-842.

10. Van Linthout S, Spillmann F, Schultheiss HP, Tschöpe C: High-density lipoprotein at the interface of type 2 diabetes mellitus and cardiovascular disorders. Curr Pharm Des 2010, 16:1504-1516.

11. McLaughlin T, Abbasi F, Cheal K, Chu J, Lamendola C, Reaven G: Use of metabolic markers to identify overweight individuals who are insulin resistant. Ann Intern Med 2003, 139:802-809. 
12. McLaughlin T, Reaven G, Abbasi F, Lamendola C, Saad M, Waters D, Simon J, Krauss RM: Is there a simple way to identify insulin-resistant individuals at increased risk of cardiovascular disease? Am J Cardiol 2005, 96:399-404.

13. Hadaegh F, Hatami M, Tohidi M, Sarbakhsh P, Saadat N, Azizi F: Lipid ratios and appropriate cut off values for prediction of diabetes: a cohort of Iranian men and women. Lipids Health Dis 2010, 9:85.

14. Kannel WB, Vasan RS, Keyes MJ, Sullivan LM, Robins SJ: Usefulness of the triglyceride-high-density lipoprotein versus the cholesterol-high-density lipoprotein ratio for predicting insulin resistance and cardiometabolic risk (from the Framingham Offspring Cohort). Am J Cardiol 2008, 101:497-501.

15. Sumner AE, Finley KB, Genovese DJ, Criqui MH, Boston RC: Fasting triglyceride and the triglyceride-HDL cholesterol ratio are not markers of insulin resistance in African Americans. Arch Intern Med 2005, 165:1395-1400.

16. Kim-Dorner SJ, Deuster PA, Zeno SA, Remaley AT, Poth M: Should triglycerides and the triglycerides to high-density lipoprotein cholesterol ratio be used as surrogates for insulin resistance? Metabolism 2010, 59:299-304.

17. Kimm H, Lee SW, Lee HS, Shim KW, Cho CY, Yun JE, Jee SH: Associations between lipid measures and metabolic syndrome, insulin resistance and adiponectin. Usefulness of lipid ratios in Korean men and women. Circ J 2010, 74:931-937.

18. Li C, Ford ES, Meng YX, Mokdad AH, Reaven GM: Does the association of the triglyceride to high-density lipoprotein cholesterol ratio with fasting serum insulin differ by race/ethnicity? Cardiovasc Diabetol 2008, 7:4.

19. Sumner AE, Harman JL, Buxbaum SG, Miller BV, Tambay AV, Wyatt SB, Taylor HA, Rotimi CN, Sarpong DF: The triglyceride/high-density lipoprotein cholesterol ratio fails to predict insulin resistance in AfricanAmerican women: an analysis of Jackson Heart Study. Metab Syndr Relat Disord 2010, 8:511-514.

20. Chien MY, Wu YT, Kwan WK, Chien CL: Reliability of the Chinese version of the 7-day recall physical activity questionnaire and its correlation with impairments of disease and function. Formosan J Phys Ther 2003, 28:1-8.

21. Hadaegh F, Khalili D, Ghasemi A, Tohidi M, Sheikholeslami F, Azizi F: Triglyceride/HDL-cholesterol ratio is an independent predictor for coronary heart disease in a population of Iranian men. Nutr Metab Cardiovasc Dis 2009, 19:401-408.

22. Tohidi M, Hatami M, Hadaegh F, Azizi F: Triglycerides and triglycerides to high-density lipoprotein cholesterol ratio are strong predictors of incident hypertension in Middle Eastern women. J Hum Hypertens 2011.

23. Hermans MP, Ahn SA, Rousseau MF: $\log (\mathrm{TG}) / \mathrm{HDL}-\mathrm{C}$ is related to both residual cardiometabolic risk and $\beta$-cell function loss in type 2 diabetes males. Cardiovasc Diabetol 2010, 9:88.

24. Li C, Ford ES, Tsai J, Zhao G, Balluz LS, Gidding SS: Serum non-high-density lipoprotein cholesterol concentration and risk of death from cardiovascular diseases among U.S. adults with diagnosed diabetes: the Third National Health and Nutrition Examination Survey linked mortality study. Cardiovasc Diabetol 2011, 10:46.

doi:10.1186/1475-2840-10-93

Cite this article as: Chiang et al:: Predicting insulin resistance using the triglyceride-to-high-density lipoprotein cholesterol ratio in Taiwanese adults. Cardiovascular Diabetology 2011 10:93.

\section{Submit your next manuscript to BioMed Central and take full advantage of:}

- Convenient online submission

- Thorough peer review

- No space constraints or color figure charges

- Immediate publication on acceptance

- Inclusion in PubMed, CAS, Scopus and Google Scholar

- Research which is freely available for redistribution

Submit your manuscript at www.biomedcentral.com/submit
Biomed Central 